Table 1. SARS-CoV-2 complementary ncRNAs and associated immunological disorders

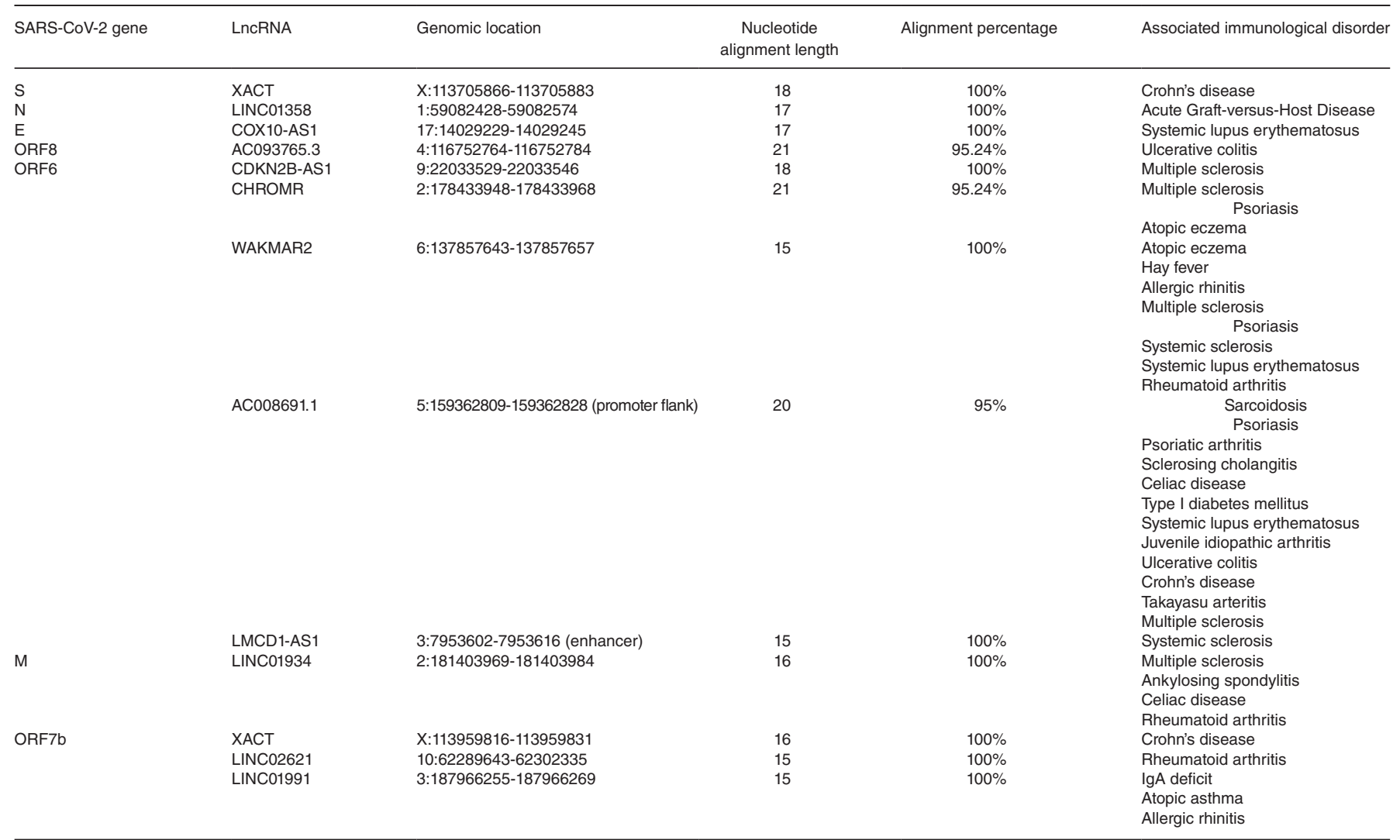

\section{POS0350 EPIONE APPLICATION: AN INTEGRATED GENOTYPE ANALYSIS WEB SERVER FOR CLINICAL GENOMICS IN SYSTEMIC LUPUS ERYTHEMATOSUS (SLE)}

L. Papageorgiou ${ }^{1}$, H. Alkenaris ${ }^{1}$, M. Zervou ${ }^{2}$, D. Vlachakis ${ }^{1}$, G. Goulielmos ${ }^{2}$, E. Eliopoulos ${ }^{1} .^{1}$ Agricultural University of Athens, Laboratory of Genetics, Department of Biotechnology, Athens, Greece; ${ }^{2}$ University of Crete, Section of Molecular Pathology and Human Genetics, Department of Internal Medicine, School of Medicine, Heraklion, Greece

Background: Genome wide association studies (GWAS) have successfully identified novel autoimmune disease-associated loci, with many of them shared by multiple disease-associated pathways but much of the genetics and pathophysiological mechanisms remain still obscure [1-3]. SLE is a chronic, highly heterogeneous autoimmune disease, characterized by differences in autoantibody profile, serum cytokines, and a multi-system involvement [4]. Epione Application is an integrated bioinformatics web-tool designed to assist medical experts and researchers in the process of diagnosing SLE [5].

Objectives: To identify the most credible gene variants and single nucleotide polymorphisms (SNPs), causing SLE using the genomic data provided for the patient and aid the medical expert in SLE diagnosis [5].

Methods: In the present study, we have analyzed more than 70.000 SLE-related publications using data mining and semantic techniques towards extracting the SLE -related genes and SNPs [6]. The extracted knowledge has been filtered, evaluated, annotated, classified, and stored in the Epione Application Database (EAD) (Figure 1). Moreover, an updated gene regulatory network with the genes implements in SLE has been estimated [7]. This was followed by the design and development of the Epione application, in which the generated datasets and results were included. The application has been tested and presented here with WES data from several related patients with SLE [8].

Results: SLE-related SNPS and variants identified in genome-wide association studies (GWAS), whole-genome (WGS), whole-exome (WES), or targeted sequencing information are classified, annotated, and analyzed in an integrated patient profile with clinical significance information. Probable genes associated with the patient's genomic profile are visualized with several graphs, including chromosome ideograms, statistic bars, and regulatory networks through data mining studies with relative publications, to obtain a representative number of the most credible candidate genes and biological pathways associated with the SLE. An evaluation study was performed on 7 patients from a three-generation family with SLE [9]. All the recognized gene variants that were previously considered SLE-associated were properly identified in the output profile per patient, and by comparing the results, new findings have emerged.
Conclusion: The Epione application was designed to assist medical doctor diagnosis from the early stages by using the patients' genomic data $[5,8,10]$. Its diagnosis-oriented output presents the patient profile through which the user is provided with a structured set of results in various categories, which are generated based on the list of the most predictable candidate gene variants related to SLE. This novel and accessible webserver tool of SLE to assist medical experts in the clinical genomics and precision medicine procedure is available at http://143.233.188.162/epione/.

REFERENCES:

[1] Molineros JE et al. (2017). Hum Mol Genet 26:1205-1216.

[2] Sciascia S et al. (2018). F1000 Res, 2018:1-17.

[3] Gonzalez-Serna D et al. (2020). Sci Rep 10:1862.

[4] Harley JB et al. (2006). Springer Semin Immun 28:119-130.

[5] Goulielmos GN et al. (2018). Gene 668:59-72.

[6] Zhao Y et al. (2020). Front Genet 11:400.

[7] Song YL and Chen S (2009). Interdiscip Sci 1:179-186.

[8] Koile D et al. (2018). BMC Bioinformatics 19:25

[9] Albertsen HM et al. (2019). Mol Med Rep 19:1716-1720.

[10] Ebrahimiyan $\mathrm{H}$ et al. (2018). Meta Gene 16:241-247.

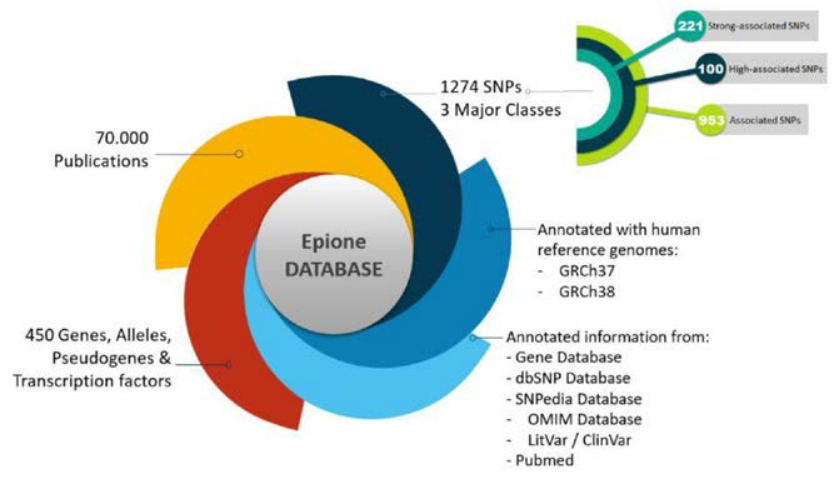

Figure 1. The Epione application database (EAD) for SLE.

Disclosure of Interests: None declared

DOI: 10.1136/annrheumdis-2021-eular.1496 